\title{
The Role of CHMP4C on Proliferation in the Human Lung Cancer A549 Cells
}

\author{
Kang Li, Jianxiang Liu, Mei Tian, Chunnan Piao, Jianlei Ruan, Ling Gao, Xuesong Qi, \\ Gang Gao, Xu Su* \\ Key Laboratory of Radiological Protection and Nuclear Emergency, China CDC, National Institute for \\ Radiological Protection, Chinese Center for Disease Control and Prevention, Beijing, China \\ Email: *suxu@nirp.cn
}

Received 17 November 2015; accepted 11 December 2015; published 14 December 2015

Copyright (C) 2015 by authors and Scientific Research Publishing Inc.

This work is licensed under the Creative Commons Attribution International License (CC BY).

http://creativecommons.org/licenses/by/4.0/

(c) (i) Open Access

\begin{abstract}
The human lung cancer has high incidence rate and mortality among the carcinoma. The research on enhancing the efficacy of therapy for lung cancer is significant. A resent research found that as a subunit of ESCRT-III, CHMP4C functioned to retard abscission timing to coordinate midbody resolution and prevent accumulation of DNA damage in the abscission checkpoint through phosphorylated by AuroraB. In the current study, we evaluated the possible mechanism of the effects of CHMP4C inhibition on cell cycle and cell survival in A549 cells. We found that CHMP4C knockdown caused lagging $S$ phase in cell cycle through enhancing the phosphorylation of $R b$, raising the expression of cyclin B1-cdc2 and suppressing the activation of cyclin A. Meanwhile, CHMP4C deletion depressed cell survival via decreasing cell viability and increasing caspase 3/7 activity. This study may promote new significant reference and advance for the mechanism underlying specific function of CHMP4C as well as further research on enhancing therapy effect on non-small lung cancer.
\end{abstract}

\section{Keywords}

The Human Lung Cancer, A549, CHMP4C, siRNA

\section{Introduction}

The human lung cancer is generally categorized into small cell lung cancer (SCLC) and non-small cell lung cancer (NSCLC) based on clinical. NSCLC accounts for 84\% in the human lung cancer, with the 5-year survival rate of approximate $16.6 \%$ [1] [2]. Hence, how to improve efficiency in diagnostics and therapy poses a critical challenge. It is essential to find an effective therapeutic target for achieving better therapy effects.

CHMP4C was found recently a critical role in the abscission timing checkpoint which governs the cytokinetic 
abscission to monitor the final separation of the two daughter cells to insure the property abscission time [3]-[5]. Besides, CHMP4C has lower expression level in normal tissues and increased expression in cancer cells. In one research, Genome wide association studies (GWAS) meta-analysis identifies three new susceptibility loci for ovarian cancer, and CHMP4C is one of them [6]. CHMP4C also plays an important role in exosome production [7] [8].

As so far, the research on CHMP4C in cancer therapy is just beginning. In the present study, we want to explore the possible mechanism of the function of CHMP4C on cell cycle and cell survival in A549 cells. This study may promote new significant reference and advance for the specific function of CHMP4C, and further research on enhancing therapeutic effect on non-small lung cancer. CHMP4C may also be a meaningful drug target gene for lung cancer therapy.

\section{Materials and Methods}

\subsection{Cell Culture}

The human lung cancer A549 cell line (Cell resource center, Peking union medical college) was cultured in RPMI-1640 containing $10 \%$ fetal bovine serum (Invitrogen, USA), incubated in $37^{\circ} \mathrm{C}$ humidified incubator with $5 \% \mathrm{CO}_{2}$.

\section{2. siRNAs Transfection}

A549 cells grew to the concentration of 80\% and were transfected with siCHMP4C (Ambion) or negative control siRNA (Ambion). All transfection of A549 cells with siRNA were performed using Lipofectamine RNAi MAX reagent. $48 \mathrm{~h}$ after transfection, the target gene knockdown efficiency was checked by western blotting, and the cells were harvested for testing.

\subsection{Western Blot}

The cells were harvested and lysed in RIPA lysis buffer (Thermo Scientific Pierce). The protein was collected at $12,000 \mathrm{~g}$ for $15 \mathrm{~min}$ at $4^{\circ} \mathrm{C}$ and measured by BCA protein assay kit (Pierce, USA). Equal amounts of protein were separated on $10 \%$ SDS-polyacrylamide gels and blotted on nitrocellulose membranes for western blot analysis. The membranes were blocked in $5 \%$ nonfat milk and then incubated with the primary antibodies: anti-CHMP4C antibody (Abcam), anti-Rb (Ser795) antibody, anti-cycinB1 antibody, anti-cdc2 (Tyr15) antibody, anti-cycinA antibody and anti $\beta$-actin antibody (Cell Signaling Technology). Membranes were washed in tris-buffered saline containing $0.5 \%$ tween- 20 and then incubated with goat anti-rabbit lgG (Cell Signaling Technology) or goat anti-mouse lgG (Cell Signaling Technology) conjugated to horseradish peroxidase for $1 \mathrm{~h}$ at room temperature. The membranes were detected using Chemiluminescence liquid (Thermo Scientific Pierce) according to the manufacturer's protocol and analyzed by the Image J software (Bio-Rad).

\subsection{Cell Cycle Assay}

Cells were harvested and treated with Vibrant Dyecycle green stain (Invitrogen, USA) at $37^{\circ} \mathrm{C}$ for $30 \mathrm{~min}$ in dark place. And then cell cycle was analyzed on a flow cytometer.

\subsection{Cell Viability Assay}

After treatment, cells were mixed with 10\% volume of alamarblue reagent (Invitrogen, USA) and incubated for $30 \mathrm{~min}$ at $37^{\circ} \mathrm{C}$, protected from direct light. Results were recorded using florescence at 570/585 nm (excitation/ emission).

\subsection{Cell Apoptosis Assay}

Cells were washed twice with cold PBS and then resuspended in $1 \times$ binding buffer (BD) at a concentration of 1 $\times 10^{6}$ cells/ml. After stained with PE AnnexinV and 7-AAD (BD), samples were analyzed by flow cytometry.

\subsection{Caspase $3 / 7$ Activity Assay}

Cells were seeded in 96 well plates at a concentration of $1 \times 10^{4}$ cells $/ \mathrm{ml}$. $48 \mathrm{~h}$ after tansfection with siCHMP4C, 
$100 \mu 1$ of Caspase-GloR 3/7 reagent (Promega) was added to each well and incubated at room temperature for 30 min, luminescence values were read out (Microplate multimode reader, Turner Biosystems).

\subsection{Statistical Analysis}

Data analysis are presented as the mean \pm SE of three independent experiments. Differences in mean values between groups were determined by students' t test using Microsoft Excel (Microsoft Campus, Redmond, WA, USA) with p-value $<0.05$ indicating statistical significance.

\section{Results}

\subsection{CHMP4C Inhibition Decreased Cell Viability and Promoted Cell Apoptosis by Enhancing Caspase 3/7 Activity}

To detect the reason for CHMP4C deletion depressing cell survival, we first employed cell viability and apoptosis assays in A549 cells with or without CHMP4C silencing. The results indicated that CHMP4C knockdown decreased cell viability and produced apoptosis compared to normal CHMP4C expression.

We next checked the activity of caspase 3/7 and then ascertained that silencing of CHMP4C increased the activity of caspase 3/7. We demonstrated that CHMP4C promoted cell apoptosis through activation of caspase 3/7 (Figure 1).

(a)

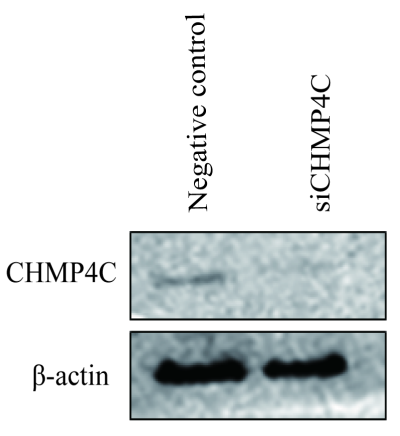

(c)
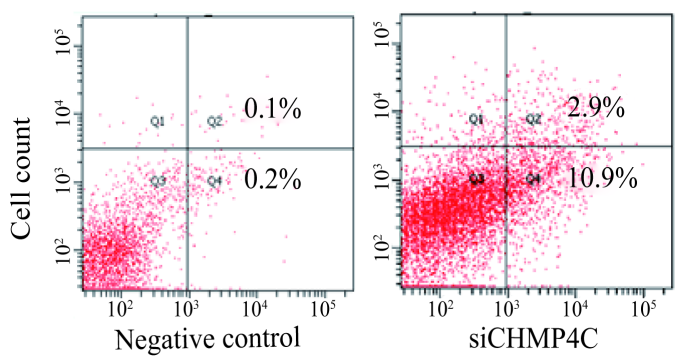

(b)

(d)
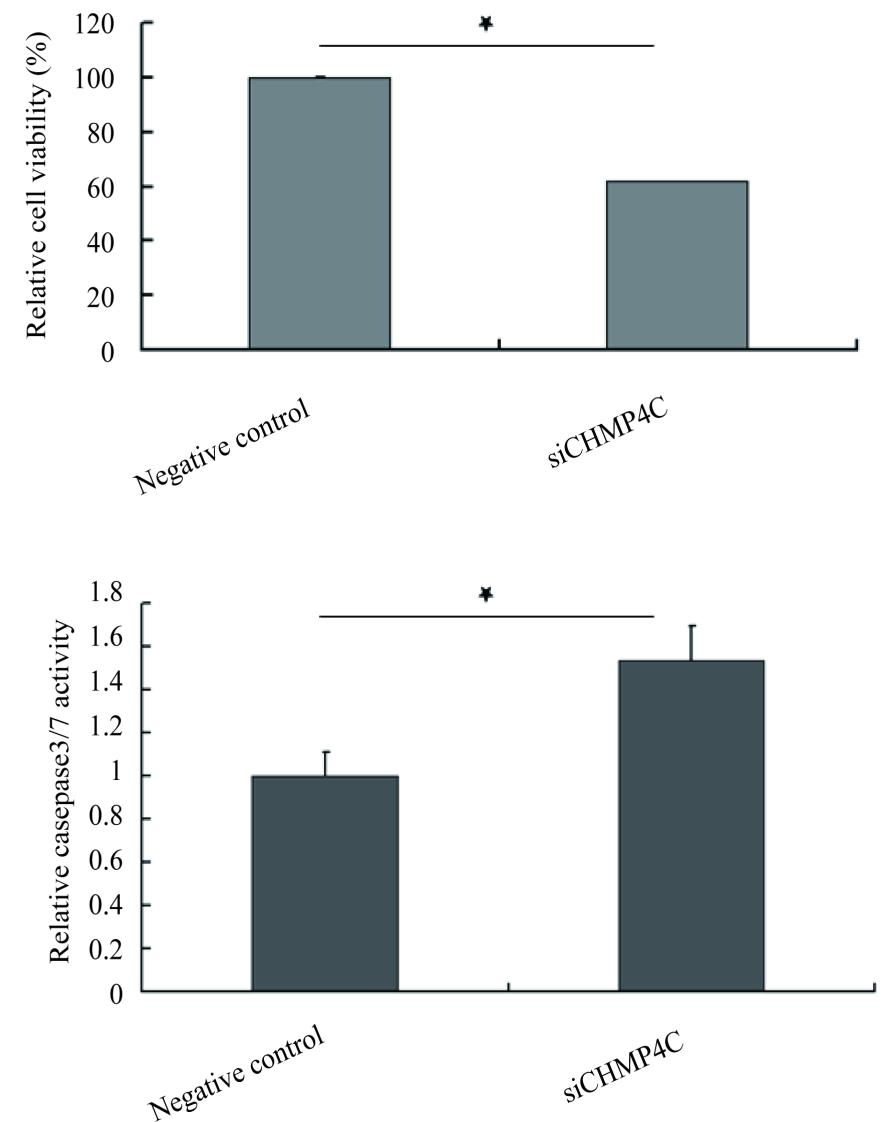

Figure 1. CHMP4C inhibition decreased cell viability and promoted cell apoptosis by enhancing caspase 3/7 activity. (a) After transfection for $48 \mathrm{~h}$, siRNAs transfection efficiency of A549 cells was assayed by western blotting; (b) The A549 cells were transfected with siCHMP4C for $48 \mathrm{~h}$. The cells were stained with alamarblue. The fluorescence intensity was read out at 570/585 nm (excitation/emission); (c) Apoptosis assay of A549 cells with or without siCHMP4C transfection; (d) Relative caspase 3/7 viability of A549 cells treated by siCHMP4C for $48 \mathrm{~h}$ was measured. Data are presented as the mean \pm SE of three independent experiments, $* \mathrm{p}<0.05$. 


\subsection{CHMP4C Silencing Retards S-Phase of the Cell Cycle through Regulation of Rb, Cyclin B1-cdc2 and Cyclin A}

From the result that CHMP4C silencing delayed S-phase in the cell cycle, we wished to explore the possible mechanism underlying CHMP4C and cell cycle. A549 cells were depleted of CHMP4C for $24 \mathrm{~h}$ and the delayed $\mathrm{S}$ phase was checked (Figure 2(a)).

And then, we analyzed cell cycle relevant protein by western bolt. Rb plays a critical role in G1/S transition, and we found CHMP4C deletion enhanced the phosphorylation of Rb in the ser795 site. Meanwhile the result also showed that inhibition of CHMP4C expression upregulated CyclinB1 and cdc2 which are major in controlling G2/M checkpoint [9] [10]. However, CyclinA was abserved downregulated by knockdown of CHMP4C, which is necessary to $\mathrm{S}$ phase progression (Figure 2(b)).

\section{Discussion}

Lung adenocarcinoma often occurs in women and non-smokers. Their five-year survival rate was only about 15\%. Chemo-radiotherapy remains the major treatment method for lung adenocarcinoma but limited success has been achieved in the clinical. With further investigation on the treatment of lung adenocarcinoma, targeted therapy has been intensively researched to improve therapy effect [11].

CHMP4C is a subunit of ESCRT-III (Endosome sorting complex-III) and plays a major role at the cytokinetic abscission checkpoint by delaying mitosis to prevent premature and accumulation of DNA damage. One recent research reported that CHMP4C is overexpressed in cancer tissues and may have relation with poor prognosis of ovarian cancer.

Current investigations on CHMP4C mainly focus on its function endosome generation [12]. But, the mechanism of its function in cellular response is not clear. In the current study, we explored the mechanism underling CHMP4C and cell survival as well as cell cycle.

In the apoptosis cysteine aspartic acid-specific protease (caspase) signaling pathway, caspase-8, caspase-9, and caspase-10 act at upstream, which couple cell death stimuli to the downstream caspase-3 and caspase- 7 . Caspase-3 and caspase-7 are both activated universally during apoptosis and play key effector roles in apoptosis

(a)

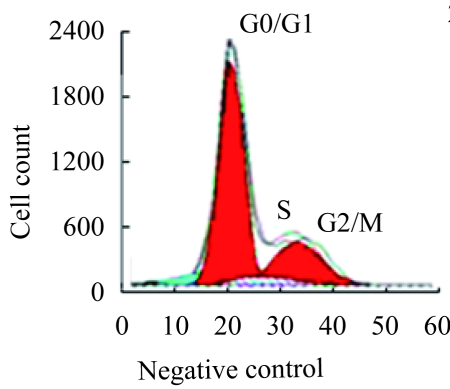

(c)

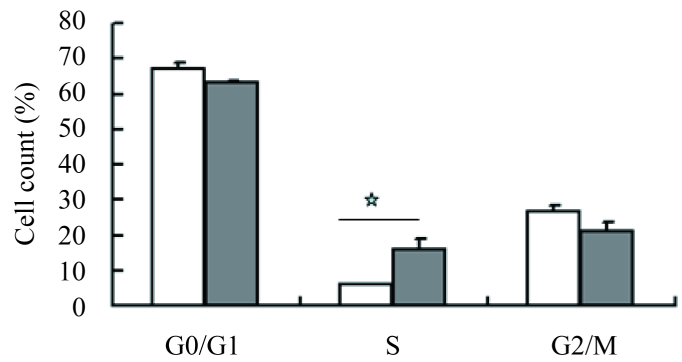

(b)
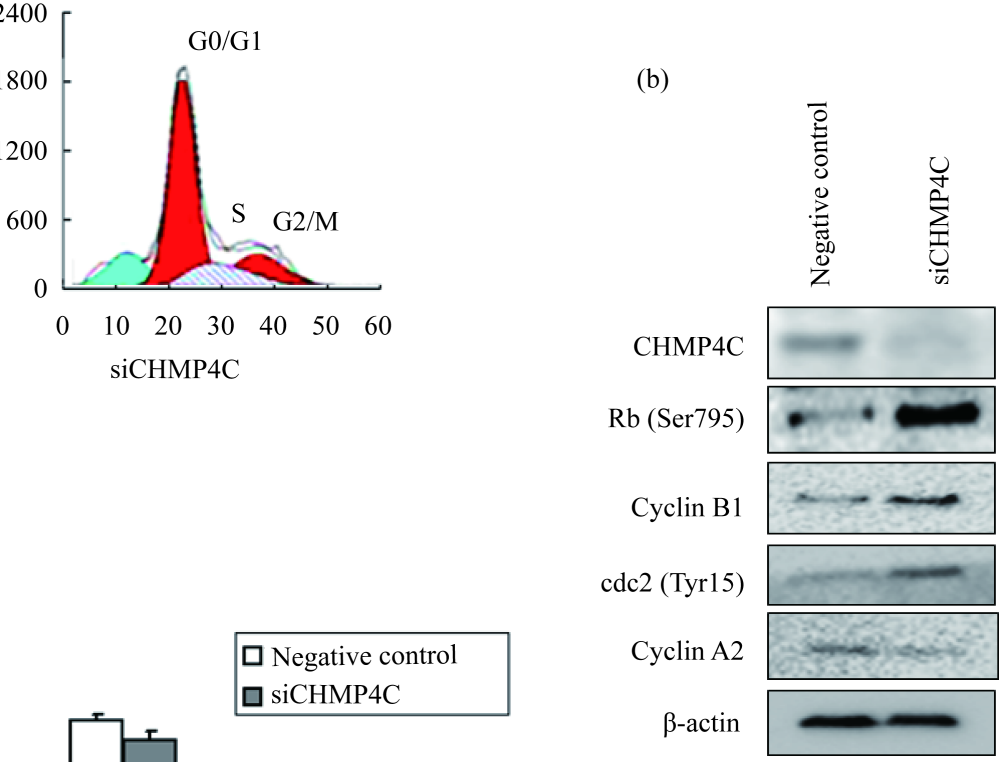

Figure 2. CHMP4C silencing retards S-phase of the cell cycle through regulation of Rb, cyclin B1-cdc2 and cyclin A. (a) Cell cycle assay for A549 cells with or without siCHMP4C transfection; (b) The A549 cells were transfected with siCHMP4C for $48 \mathrm{~h}$. The cell lysates were analyzed by western blot using the indicated antibodies; (c) Quantification of cell cycle assay. Data are presented as the mean \pm SE of three independent experiments, $* p<0.05$. 
in mammalian cells [13]. Our results demonstrated that inhibition of CHMP4C promoted cell apoptosis via enhancing the activity of caspase-3 and caspase-7.

$\mathrm{Rb}$ (Retinoblastoma gene) regulates cell proliferation by governing G1/S transition in the cell cycle [14]. The cyclinB1-cdc2 kinase is critical in regulating the G2/M transition. Overexpression of cyclinB1-cdc2 can promote G2/M transition [15] [16]. Cyclin A is associated with the E2F transcription factor control and is required for S-phase in cell cycle. Inhibition of cyclin A can delay the G1 to S transition and prevent DNA replication [17]. In the study, we found that CHMP4C depletion enhanced Rb phosphorylation, increased cyclinB1-cdc2 expression and reduced cyclin A expression, which in turn leaded to lagging S-phase.

\section{Conclusion}

In conclusion, our results further reveal that CHMP4C deficiency disturbed cell cycle progress and enhanced cell death as well as its mechanism, suggesting a new therapy target for the treatment of lung cancer.

\section{References}

[1] Nguyen, K.S., Neal, J.W. and Wakelee, H. (2014) Review of the Current Targeted Therapies for Non-Small-Cell Lung Cancer. World Journal of Clinical Oncology, 5, 576-587. http://dx.doi.org/10.5306/wjco.v5.i4.576

[2] Liu, Y.-T., Hao, X.-Z., Li, J.-L., Hu, X.-S., Wang, Y., Wang, Z.-P. and Shi, Y.-K. (2015) Survival of Patients with Advanced Lung Adenocarcinoma before and after Approved Use of Gefitinib in China. Thoracic Cancer, 6, 636-642. http://dx.doi.org/10.1111/1759-7714.12267

[3] Carlton, J.G., Caballe, A., Agromayor, M., Kloc, M. and Martin-Serrano, J. (2012) ESCRT-III Governs the Aurora B-Mediated Abscission Checkpoint through CHMP4C. Science, 336, 220-225. http://dx.doi.org/10.1126/science.1217180

[4] Jimenez, A.J., Maiuri, P., Lafaurie-Janvore, J., Divoux, S., Piel, M. and Perez, F. (2014) ESCRT Machinery Is Required for Plasma Membrane Repair. Science, 343, 1247136. http://dx.doi.org/10.1126/science.1247136

[5] Lafaurie-Janvore, J., Maiuri, P., Wang, I., Pinot, M., Manneville, J.-B., Betz, T. and Piel, M. (2013) ESCRT-III Assembly and Cytokinetic Abscission Are Induced by Tension Release in the Intercellular Bridge. Science, 339, 16251629. http://dx.doi.org/10.1126/science.1233866

[6] Pharoah, P.D.P., Tsai, Y.-Y., Ramus, S.J., Phelan, C.M., Goode, E.L., Lawrenson, K. and Sellers, T.A. (2013) GWAS Meta-Analysis and Replication Identifies Three New Susceptibility Loci for Ovarian Cancer. Nature Genetics, 4, 362370. http://dx.doi.org/10.1038/ng.2564

[7] van der Waal, M.S., Hengeveld, R.C.C., van der Horst, A. and Lens, S.M.A. (2012) Cell Division Control by the Chromosomal Passenger Complex. Experimental Cell Research, 318, 1407-1420. http://dx.doi.org/10.1016/j.yexcr.2012.03.015

[8] Feng, Z. (2010) p53 Regulation of the IGF-1/AKT/mTOR Pathways and the Endosomal Compartment. Cold Spring Harbor Perspectives in Biology, 2, a001057. http://dx.doi.org/10.1101/cshperspect.a001057

[9] Lim, S. and Kaldis, P. (2013) Cdks, Cyclins and CKIs: Roles Beyond Cell Cycle Regulation. Development, 140, 3079-3093. http://dx.doi.org/10.1242/dev.091744

[10] Liu, P., Slater, D.M., Lenburg, M., Nevis, K., Cook, J.G. and Vaziri, C. (2009) Replication Licensing Promotes Cyclin D1 Expression and G(1) Progression in Untransformed Human Cells. Cell Cycle (Georgetown, Tex), 8, 125-136. http://dx.doi.org/10.4161/cc.8.1.7528

[11] Gower, A., Wang, Y. and Giaccone, G. (2014) Oncogenic Drivers, Targeted Therapies, and Acquired Resistance in Non-Small-Cell Lung Cancer. Journal of Molecular Medicine (Berlin), 92, 697-707. http://dx.doi.org/10.1007/s00109-014-1165-y

[12] Yu, X., Riley, T. and Levine, A.J. (2009) The Regulation of the Endosomal Compartment by p53 the Tumor Suppressor Gene. FEBS Journal, 276, 2201-2212. http://dx.doi.org/10.1111/j.1742-4658.2009.06949.x

[13] Walsh, J.G., Cullen, S.P., Sheridan, C., Lüthi, A.U., Gerner, C. and Martin, S.J. (2008) Executioner Caspase-3 and Caspase-7 Are Functionally Distinct Proteases. Proceedings of the National Academy of Sciences of the United States of America, 105, 12815-12819. http://dx.doi.org/10.1073/pnas.0707715105

[14] Yoon, I., Chung, J., Hahm, S., Park, M., Lee, Y., Ko, S. and Han, Y. (2011) Ribosomal Protein S3 Is Phosphorylated by Cdk1/cdc2 during G2/M Phase. BMB Reports, 44, 529-534. http://dx.doi.org/10.5483/BMBRep.2011.44.8.529

[15] Tsuchiya, Y., Murai, S. and Yamashita, S. (2015) Dual Inhibition of Cdc2 Protein Kinase Activation during Apoptosis in Xenopus Egg Extracts. FEBS Journal, 282, 1256-1270. http://dx.doi.org/10.1111/febs.13217 
[16] Lee, M., Cho, Y., Jung, B., Kim, S., Kang, Y., Pan, C. and Kim, Y. (2015) Parkin Induces G2/M Cell Cycle Arrest in TNF- $\alpha$-Treated HeLa Cells. Biochem. Biochemical and Biophysical Research Communications, 464, 63-69. http://dx.doi.org/10.1016/j.bbrc.2015.05.101

[17] Lapenna, S. and Giordano, A. (2009) Cell Cycle Kinases as Therapeutic Targets for Cancer. Nature Reviews Drug Discovery, 8, 547-566. http://dx.doi.org/10.1038/nrd2907 